[Vicino Oriente XVIII (2014), pp. 15-24]

\title{
GLI ITTITI E LA LORO RISCOPERTA NELLA TURCHIA REPUBBLICANA
}

\author{
Rita Francia - Sapienza Università di Roma*
}

Among the reforms of Mustafà Kemal Atatürk in Turkey, there was also the cultural one and the re-evaluation of the past. The studies of the pre-classical history and the ancient cultures, particularly the Hittites, received a substantial boost.

Keywords: Turkey; Atatürk; Hittitology, cultural reform; re-evaluation of the past

Uno degli aspetti della radicale opera di riforme messa in atto in Turchia da Mustafà Kemal, appellato poi Atatürk, nel periodo che va dal 1923 al 1938, riguardò l'ambito culturale $^{1}$. Tra tutti i settori del sapere, un'attenzione particolare fu riservata agli studi di carattere storico-archeologico e linguistico, ritenuti essere l'appendice positivista della storia, e dunque utili a dimostrare scientificamente, con prove inconfutabili, quanto l'ideologia kemalista andava diffondendo.

Nell'ultimo periodo dell'Impero Ottomano le discipline storico-archeologiche in generale, relative all'Anatolia in particolare, erano state messe da parte, nonostante fosse palese l'interesse di alcuni governi stranieri ai siti archeologici turchi e venissero avviati scavi di notevole importanza in Anatolia, come quello di Boğazköy/Hattuša nel 1906. Con l'ascesa al governo di Mustafà Kemal, questa branca del sapere venne profondamente rivalutata. La visione che lo statista aveva al riguardo emerge chiaramente da un telegramma inviato il 22 marzo 1931 all'allora primo ministro İsmet İnönü: «Le opere di grande e incomparabile valore appartenute alle civiltà ormai scomparse, che sono sepolte pressoché in tutte le regioni del nostro Paese, dovranno essere riportate alla luce, protette e classificate scientificamente in un prossimo futuro. Quanto ai monumenti che hanno sofferto della negligenza del passato, dovrebbero essere protetti con maggiore attenzione e, a tal fine, si dovrebbero formare più archeologi» ${ }^{2}$. Espressione di questo preciso volere è anche il decalogo che Atatürk dettò nel 1935 ad Afet İnan, sua figlia adottiva e personaggio chiave nella nascente Repubblica, e all'allora direttore della Türk Tarih Kurumu, "Società Turca per gli Studi Storici”, Hasan Cemil Çambel ${ }^{3}$.

* Una forma brevis di questo contributo è stata oggetto di una relazione tenuta al Center Euroasian Studies (Centro CEMAS Università di Roma «Sapienza») - Bahçeşehir (University of Istanbul) (Cse BAU) nell'aprile del 2014, per il cui interessamento ringrazio il Prof. F.L. Grassi, direttore dell'Istituzione.

1 In generale su Atatürk si veda Grassi 2008.

2 Özgüç 1982, 6.

3 Özgüç 1982, 6-7. In questo decalogo sono contemplati tutti gli aspetti della valorizzazione e tutela delle antichità, dalle testimonianze scritte ai monumenti, sotto ogni punto di vista: dalla protezione contro vandalismi, a quella contro il deterioramento provocato dagli agenti naturali; i finanziamenti che devono arrivare dalle fonti governative; la necessità di sensibilizzare la popolazione all'importanza dei reperti archeologici; il progetto di fare di Ankara, Istanbul, Bursa, Izmir ed Edirne 'centri di antichità e di monumenti storici' ad alto livello, per citarne solo alcuni. 
L'intento di Atatürk, formatosi culturalmente all'epoca riformista e progressista delle Tanzimat "riforme" 4 , fu: dare solide basi culturali alla giovane Repubblica, rivalutando il passato più remoto, per giustificare sia l'unità nazionale presente che la forza stessa dell'etnia, se non 'razza', turca'.

\section{I PRECETTI DELL'IDEOLOGIA KEMALISTA}

Il nazionalismo kemalista fondava tutta la sua dottrina su sei precetti, elencati dallo stesso Atatürk ad A. İnan: unità politica, linguistica, geografica, genealogica, vincoli storici e vincoli morali ${ }^{6}$. La visione politica del fondatore della Turchia moderna tenne dunque le dovute distanze sia dal concetto di religione, che non avrà mai parte nell'ideologia laica kemalista, sia da quello di etnia, che invece verrà riportato in auge in un secondo momento per fronteggiare questioni pratiche.

L'esclusione del concetto di etnia nelle fasi iniziali dell'affermazione della politica del nuovo governo si rivelò estremamente funzionale: permise di accogliere nella Repubblica Turca chiunque, a qualsiasi etnia appartenesse, purché turcofono. E' pur vero che dopo il Trattato di Losanna del 1923 i musulmani turchi erano nettamente dominanti, costituendo il 97\% del totale degli abitanti, ma non si potevano escludere i membri di altre etnie che pur risiedevano sul suolo turco, quali Armeni, Greci e Curdi. Per essere Turco, per appartenere cioè al nuovo stato, era necessario identificarsi in un gruppo politico-sociale che si riconosceva innanzitutto in una lingua comune. D'altra parte lo stesso statista sottolineò a più riprese l'inscindibile legame che vi era, dalla suo punto di vista, tra lingua e cultura turca: in un discorso del 1930 rimarcò che la lingua era senza dubbio l'elemento di coesione ed identificazione più importante in una nazione, pertanto coloro che si dichiaravano Turchi dovevano innanzitutto parlare il turco ${ }^{7}$. La lingua fu pertanto utilizzata quale strumento per inglobare le minoranze e a tal fine si attuarono numerose riforme, come l'eliminazione dall'insegnamento scolastico della lingua persiana e di quella araba, in favore del solo turco, e l'adozione dell'alfabeto latino per la sua scrittura.

La creazione di una solida coscienza nazionale fu una delle priorità del nuovo governo. Era infatti indispensabile che gli abitanti della nuova Turchia ritrovassero le loro radici in un passato nobile e glorioso in cui tutti potessero riconoscersi; per questo, tra tutte le scienze, quelle storico-archeologiche e linguistiche furono ampiamente rivalutate e fortemente patrocinate.

4 Questo termine è da intendersi 'riforme' sia per traduzione letterale, sia quale denominazione del periodo storico riformatore e progressista cominciato nel 1839. In questi anni vennero attuate una serie di riforme prendendo a modello la legislazione francese e in particolare le idee progressiste di Montesquieu. Le innovazioni di questo periodo servirono come prodromo per la Repubblica Turca; si veda Losano 2012, in particolare $\S 1$ e nota 2 . Per le tematiche generali affrontate in questo contributo, rimandiamo anche ad Atakuman 2008.

5 Sui concetti di nazionalismo turco, razza, e sui movimenti politico-culturali ad essi collegati: Losano 2012. Il sentimento nazionalistico che spinse Atatürk ad amare l'archeologia fu notevole, tanto che Özgüç $(1982,8)$ afferma che «è difficile separare Atatürk dall'archeologia in Turchia».

6 İnan $1930 \mathrm{~b}, 13$.

7 Arar 1981, 23-24. 


\section{IL TURAN, IL TURANISMO E IL TURCHISMO}

Nell'ambito della rivalutazione del passato, vennero ripresi e riconsiderati gli ideali del Turk Ocalari ('Focolari Turchi'), movimento fondato nel 1911-1912, con il fine di dare nuovo vigore all'ideale di unità del popolo turco. A questo movimento si deve anche la diffusione del concetto di Turan quale patria ideale da cui avrebbero avuto origine i Turchi $^{8}$. Nell'ottobre del 1935, per volere dello stesso Atatürk, venne costituita la Türk Tarih Kurumu "Società Turca per gli Studi Storici", sviluppo del Türk Tarihi Tetkik Cemiyeti, "Società Turca per le Ricerche Storiche", la cui espressione era la rivista Belleten, istituita nel 1930, sempre per interesse diretto dello statista. Molti dei membri di questa istituzione erano importanti personalità della cultura e della politica, solo per citarne alcuni: A. İnan, H.C. Çambel, Reşit Galip, Yusuf Ziya Özer, tutti, peraltro, amici personali di Atatürk. Quale supporto indispensabile alla Türk Tarih Kurumu, nel 1932, sempre su esortazione dello statista, venne fondata la Türk Dili Tetkik Cemiyeti "Società Turca per la Ricerca Linguistica", con il compito di dimostrare attraverso studi comparati l'importanza del turco tra tutte le altre lingue del mondo ${ }^{9}$.

Il riconoscere un passato comune a cui appellarsi non fu dunque un'idea originale di Atatürk: già in età ottomana, all'epoca delle Tanzimat, vi erano stati movimenti nazionalistici, come il citato Turk Ocalari, che inneggiavano ad una unità pre-ottomana del popolo turco nel mitico territorio del Turan. E' questo un leggendario paese collocato nell'Asia Centrale, considerato la culla delle popolazioni che, spinte da calamità naturali, in ondate migratorie diverse, avrebbero dato vita alle più grandi civiltà del passato preclassico. Il nazionalismo di età ottomana, che si appellava 'Turanismo' o 'Panturanismo', si riprometteva di unificare un territorio di vaste proporzioni, dall'area egea alla Cina, dall'India all'altopiano asiatico, ex Unione Sovietica, abbracciando anche l'Ungheria, la Finlandia, l'Estonia e la Lituania. Distinto dal 'Turanismo' o 'Panturanismo', almeno in origine, fu un altro movimento, il 'Turchismo' o 'Panturchismo'. Lo scopo del Turchismo era unificare sotto un'unica entità politica le popolazioni che si riconoscevano turche, anche se non risiedenti entro i confini dell'impero ottomano, come i Turchi della Russia meridionale o dell'Asia centrale. Successivamente si verificò una sovrapposizione

8 Con il termine Turan si alludeva ad un mitico territorio collocato in Asia Centrale. Il toponimo rimandava più ad una condizione ideologica che ad un'area geograficamente individuabile: una sorta di Eden, identificato solo in un secondo momento con il bassopiano turanico, tra l'Asia Centrale, il Turkmenistan, l'Uzbekistan e il Kazakistan. Originariamente il nome persiano Turan 'Terra dei Tur' abbracciava tutta l'area dell'Asia Centrale, senza chiari confini geografici. Secondo la leggenda, il popolo che l'abitava erano i Tura o Turiya, tribù nomade, noto fin dall'Avesta. La cultura iranica ha utilizzato in termine 'turanico' per indicare tutto ciò che non era iranico e l'identificazione dei Tura con i Turchi avvenne solo per questioni di assonanza: la somiglianza fonica dei due nomi favorì la sovrapposizione. Le identificazioni storiche sono ben diverse: probabilmente i Tura erano tribù appartenenti agli Sciiti ed ai Cimmeri. Per avvalorare l'identificazione dei Tura con i Turchi si ricorse anche allo Shahnameh, "Nomi dei re", opera del poeta iranico Ferdowsi, vissuto intorno al 1000 d.C., sebbene nel poema non si faccia mai alcun riferimento esplicito ad un legame tra i Tura e i Turchi. Secondo questo racconto leggendario, che ricostruisce la storia dalla creazione del mondo alla colonizzazione islamica, il figlio dell'imperatore Fereyudun si chiamava Tur/Turaj, riconosciuto successivamente come uno dei progenitori dei Turchi.

9 Gli studi condotti dalla Türk Tarih Kurumu furono resi pubblici in due congressi, nel 1932 e nel 1937; almeno tre furono i congressi furono tenuti dalla Türk Dili Tetkik Cemiyeti. 
semantica: i termini 'Turanismo' e 'Panturanismo', 'Turchismo' e 'Panturchismo' furono usati come sinonimi, pur avendo nella loro genesi origini e finalità diverse $\mathrm{e}^{10}$.

La politica di rivalutazione del passato preclassico promossa da Atatürk era basata su due teorie: i Turchi avevano abitato la Turchia da tempo immemorabile e la loro origine, da ricercare nell'area del Turan, era comune a quella di altre popolazione occidentali di stirpe indo-europea ${ }^{11}$. Infatti, in epoca preistorica la popolazione del Turan, brachicefala ${ }^{12}$, che già aveva scoperto l'allevamento, l'agricoltura, la lavorazione dei metalli, fu costretta a lasciare la sua sede originaria. Dall'Asia Centrale questa popolazione si diramò in tutte le direzioni, tanto ad est (Cina, India), quanto a ovest. Il gruppo che migrò verso ovest si fermò in Anatolia che divenne così la culla della civiltà occidentale ${ }^{13}$. Da qui gruppi migratori partirono a più riprese verso ovest e verso sud-est, dando origine alle civiltà preclassiche: la sumerica, la mesopotamica, l'egea, l'egiziana, l'italica ${ }^{14}$.

Tra i sostenitori e teorici di questa ideologia vi fu anche H.C. Çambel, padre di Halet Çambel, che ricopriva l'incarico istituzionale di addetto militare del governo in Germania. Questi era amico personale di Atatürk ed era stato membro attivo nella fondazione della Repubblica. In un suo scritto a sostegno delle idee kemaliste, affermò che gli Egei e gli Ittiti, che avevano colonizzato l' Asia Minore, nonché gli Sciiti, i Sumeri, gli Egiziani erano tutte popolazioni strettamente legate tra loro perché discendevano da un unico ceppo comune: quello dei popoli originari delle miniere di ferro degli Altai ${ }^{15}$.

\section{GLI ITTITI PROGENITORI DEI TURCHI MODERNI}

A supportare e provare con dati scientifici incontrovertibili questa teoria fu dedicata la tesi di dottorato di A. İnan, L'Anatolie le pays de la 'Race' Turque, Publication de la Faculté des Sciences Économiques et Sociales de l'Université de Genève, Genève 1941, svolta presso la cattedra del Prof. Eugen Pittard a Ginevra e poi pubblicata a cura della stessa Università. İnan fu tra i membri fondatori della Türk Tarihi Tetkik Cemiyeti "Società Turca per le Ricerche Storiche", organo che si occupava del fondamento teorico e storico delle ideologie promosse. Per la sua ricerca İnan utilizzò metodologie antropometriche, del tutto pionieristiche per l'epoca e, attraverso le misurazioni di 64.000 crani di individui

10 Si veda Losano 2012, $\S \S 2-3$.

11 Erano questi ideali circolanti fin dal XIX sec. nell'ambito del Turanismo. Tra gli autori di opere che contribuirono a fondare e diffondere queste posizioni ricordiamo: C. Bosenski (1869); L. Cahun (1896), le prime poesie nazionaliste di M. Emīn; le opere di A. Vámbéry, nonché il principale teorico dell'ideologia nazionalista turca, Ziyā Gök Alp, fervente panturchista, formatosi sull'opera di Cahun: egli affermò in una sua poesia: «la patria per i Turchi non è la Turchia né il Turkestan, la patria è un paese grande ed eterno, il Tūrān». Dopo la Prima Guerra Mondiale questi ideali furono portata avanti dall'associazione Türk Ogiāghi 'Il Focolare turco' fondata nel 1912, con la rivista Türk Yurdu.

12 Particolare rilevante per l'epoca in cui, tra le varie peculiarità proprie della razza indo-europea, si riconosceva il brachicefalismo.

13 Anche questo ideale era già circolante, promosso dal movimento denominato Anadolugiuluk 'Anatolismo', nato ad opera di giovani universitari riunitisi intorno alla rivista Anadolu Mecmuasi, ma che ebbe vita breve: dal 1924 al 1925.

14 Queste teorie furono esposte in forma canonica nell'opera di İnan 1930a, da cui fu poi ricavata una forma brevis, İnan 1931

15 Çambel 1932, 201. 
provenienti dall'Anatolia e dalla Tracia e la successiva comparazione con le misure dei crani Ittiti, arrivò a dimostrare che l'Anatolia era stata da sempre la patria di genti di razza turca brachicefala.

Stando a questi studi, pertanto, gli Ittiti potevano ritenersi i progenitori dei Turchi moderni sulla base di inconfutabili prove scientifiche. İnan ottenne il titolo di Dottore di Ricerca in Sociologia nel 1939 e poi una cattedra all'Università Ankara nel 1950.

Le tesi di İnan ben si conciliavano con quanto già da qualche decennio era stato provato da altri studiosi, primo tra tutti Bedřich Hrozný, del tutto estranei alla causa turca, cioè che gli Ittiti parlavano una lingua indo-europea ${ }^{16}$. Da queste premesse si comprende il motivo del grande interesse di Atatürk e dei suoi collaboratori per questa civiltà e la sua storia: tra tutte le popolazioni preclassiche che si erano succedute sul suolo anatolico, agli Ittiti fu riservato un posto d'onore, poiché potevano essere considerati l'anello di congiunzione tra le popolazioni turaniche di epoca pre-storica e i Turchi di epoca moderna, di cui erano ritenuti essere diretti progenitori.

La propaganda politica dunque utilizzò dati scientifici certi, quali la matrice indoeuropea, l'appartenenza alla razza brachicefala degli Ittiti e il fatto che si fossero stanziati in Anatolia, mescolandoli alle notizie pseudo-storiche di derivazione epico-letteraria, come l'ipotetica migrazione dal mitico Turan. Dalla fusione di questi elementi, nacque un assioma che rispondeva pienamente alle finalità del governo: gli Ittiti erano a tutti gli effetti da ritenersi una popolazione anatolica, dunque turca, e da essi discendevano direttamente i turchi moderni. Ben si comprende dunque l'appoggio incondizionato del governo agli scavi archeologici di siti preclassici come Alacahöyük, Ahlatlıbel, Karalar, Göllüdağ, Boghazköy/Hattuša, come è provato dalle concessioni di scavo che Kurt Bittel, direttore della missione archeologica a Hattuša, ottenne incondizionatamente in quegli anni: da questi siti, che rappresentavano l'espressione diretta delle civiltà da cui poi erano discesi i Turchi moderni, potevano venire prove inconfutabili a sostegno delle tesi divulgate.

L'allora direttore di scavo di Alacahöyük, Hamit Zübeyr Koşay ${ }^{17}$, nel presentare il contributo di Alacahöyük alla storia della civiltà, affermò in un suo scritto che la cultura dell'età del rame di Alaca presentava connessioni sia con le culture pre-sciite della Russia meridionale e dell'area del Kuban, sia con quella dei Sumeri per la somiglianza delle tombe reali di Ur con quelle lì rinvenute. Queste affinità a suo avviso erano imputabili alla comune origine derivante dalle migrazioni dei popoli che dall'Asia Centrale si erano diramati ovunque: dalla Cina alla Scandinavia. L'antica cultura di Alacahöyük, sosteneva Koşay, era strettamente imparentata in tutto e per tutto con quella turca, essendone la diretta antecedente. Era inoltre innegabile l'apporto che la razza turca aveva dato all'umanità: essa era stata fondamentale per la nascita, la diffusione e il progresso della civiltà, tanto in età preistorica che storica ${ }^{18}$.

6 Hrozný 1915; 1917.

17 Originario di un paesino degli Urali, messosi in luce per le sue doti scientifiche, fu sostenuto negli studi dal governo dapprima in Turchia e poi in Ungheria. Una volta tornato in Turchia, divenne Ministro dell'Educazione e successivamente direttore del Museo Etnografico di Ankara. Dall'alto della sua posizione in ambito politico, supportò sempre, dal 1931 in poi, lo scavo di Boghazköy/Hattuša.

18 Koşay 1943, 31-32. 


\section{LA TEORIA LINGUISTICA}

Come abbiamo già accennato, uno dei punti di forza dello storicismo kemalista trovava fondamento nelle teorie linguistiche. Tra le tesi diffuse vi fu anche quella denominata Güneş Dil Teorisi "Teoria della Lingua del Sole", secondo cui il turco era l'idioma più vicino a quello da cui tutti gli altri avevano avuto origine, la 'Lingua del Sole', appunto, parlata nel mitico Turan. Y.Z. Özer, di professione avvocato, membro attivo della Società Turca per la Ricerca Storica, in uno studio presentato al primo congresso della Società nel 1932, arrivò ad affermare la seguente teoria: quando le popolazioni del Turan, costituite da tribù sia ariane che semitiche, lasciarono la loro sede originaria in epoca neolitica, erano tutte parlanti turco; separatisi poi per fattori esterni, culturali, climatici etc., ogni popolo sviluppò un idioma diverso, ma la lingua d'origine restava il turco, come era dimostrato dalle connessioni esistenti tra le lingue semitiche e quelle arie. Da ciò derivava anche che $\mathrm{i}$ Turchi, da sempre, avevano parlato il turco e da sempre erano stati Turchi ${ }^{19}$.

\section{L'ISTITUZIONE DI NUOVE CATTEDRE E LA FORMAZIONE DI GIOVANI STUDIOSI TURCHI}

Tra i meriti che possiamo riconoscere al processo di rivalutazione del passato preclassico kemalista, oltre al rilascio delle concessioni di scavo, vi fu l'istituzione di nuovi insegnamenti umanistici nelle Università di Istanbul ed Ankara. In questi Atenei vennero istituite le cattedre di Ittito, Sumerico, Cinese, Ungherese, Latino, Linguistica, Geografia, Storia, lingue e culture con cui i Turchi erano entrati in qualche modo in contatto nel corso della storia. Tra i giovani cultori di queste discipline vi furono quelli che poi diventeranno note personalità nell'ambito degli studi ittitologici, come ad esempio: Tahsin Özgüç, Nimet Dinçer-Özgüç, Mustafà Kalaç.

In questa politica culturale, Atatürk fu lungimirante e desiderò dare alla Turchia studiosi di alto livello internazionale nelle discipline storico-archeologiche e per questo inviò all'estero giovani promettenti, affinché approfondissero la loro preparazione, imparassero lingue straniere e nuove metodologie di ricerca cosicché, una volta tornati, potessero valorizzare i beni storico archeologici della madrepatria. Tra questi giovani vi furono lo stesso Koşay, oltre a Remzi Oğuz Arık, che fu il primo archeologo turco e scavò anche ad Alacahöyük insieme a Koşay e Sedat Alp.

Analogamente, lo statista fu propenso ad invitare professori stranieri nelle maggiori Università turche per promuovere gli insegnamenti delle discipline storico-archeologiche ${ }^{20}$. A tal fine anche la Turchia, come gli Stati Uniti, si mostrò propensa ad accogliere gli studiosi tedeschi di origine ebraica costretti a lasciare il loro paese a causa delle persecuzioni razziali. Secondo Atatürk, infatti, proprio sotto l'egida della comune origine delle razze, i popoli dovevano trovare la motivazione per riunirsi, pur mantenendo la propria identità culturale, politica e nazionale.

Fu così che studiosi come Benno Landsberger, Hans Gustav Güterbock, ebreo da parte di padre, ottennero una cattedra all'università di Ankara dove avviarono gli insegnamenti di

19 Özer 1932, 246.

20 Özgüç 1982, 7-8. 
Assiriologia e Ittitologia ${ }^{21}$. In questo periodo ad Ankara si formò quel gruppo di giovani ed entusiasti studiosi che Sedat Alp definì «Çivi Yazısı İlmi’nin Ankara'daki Kurucuları» «i fondatori dello studio del cuneiforme ad Ankara». Costui, come abbiamo già accennato, fu trai giovani brillanti inviato in Germania dal governo ed è interessante seguire le vicende della sua vita in quegli anni, perché strettamente legate a quelle che favorirono lo sviluppo e l'affermarsi degli studi ittitologici per diretto interesse del governo turco.

\section{Il soggiorno in Germania di Sedat Alp E L'AfFermazione dell'ItTitologia in TURCHIA}

Il giovane Alp fu mandato in Germania nel 1932 per approfondire le discipline storicoarcheologiche. Dapprima egli si dedicò allo studio del tedesco presso un liceo di provincia, imparando mediamente duecento parole al giorno, e successivamente, tra il 1933-1934, su diretto invito del ministro turco dell'Educazione, Reşit Galip, si trasferì a Berlino per l'apprendimento dell'Archeologia e della Storia antica. Qualche tempo dopo essersi stabilito nella capitale tedesca, Alp assistette ad una conferenza di Fritz Schachermeyr sugli Ittiti e sugli Akkadi. Rimasto affascinato da queste popolazioni e dalla loro storia, il giovane manifestò il desiderio studiare Ittitologia. Dopo aver ricevuto il permesso dal suo governo, si recò a Lipsia dove fu per molto tempo l'unico studente di Johannes Friedrich; contemporaneamente studiò Assiriologia con B. Landsberger. In quegli anni il governo turco inviava ai giovani all'estero il giornale 'Ulus' e fu proprio da questa fonte che Alp venne a conoscenza della nuova facoltà di Lingue, Storia, Geografia che si andava istituendo ad Ankara. Preso contatto con l'allora Ispettore Ministeriale di Berlino, Cevat Dursunoğlu, avanzò la proposta di chiamare ad Ankara, per la cattedra di Assiriologia, B. Landsberger che, essendo ebreo, era stato licenziato dall'Università di Lipsia ed era rimasto senza lavoro. La richiesta di Alp venne accolta di buon grado dal governo e così nel 1936 Landsberger venne invitato in Turchia a ricoprire l'insegnamento di Assiriologia ad Ankara, proposta che il professore tedesco accettò senza esitazioni. Era interesse della Turchia che i professori invitati ad insegnare nei propri Atenei avessero a disposizione tutto quanto fosse loro necessario per svolgere al meglio il loro mandato, pertanto quando Landsberger espresse il desiderio di avere con sé la biblioteca che fu di Heinrich Zimmer, suo professore, venne subito accontentato.

Al seguito di Lansberger giunse ad Ankara anche Hans Gustav Güterbock, che all'epoca aveva 28 anni, e stava conducendo presso di lui un dottorato in Assiriologia e Ittitologia. Come Landsberger, anche Güterbock era stato costretto a lasciare la Germania per motivi razziali. L'arrivo del giovane studioso destò sorpresa e interesse tra gli allievi, che non si aspettavano di incontrare un così giovane e fascinoso professore. Oltre che per la sua competenza nella disciplina, Güterbock fu apprezzato anche per le doti umane di tolleranza e di pazienza riguardo all'insegnamento stesso. Il giovane tedesco imparò subito la lingua turca e restò colpito dall'entusiasmo e dal desiderio di apprendere degli studenti, tanto da dire in seguito di non aver più avuto un simile gruppo di allievi; dal 1933 al 1936 Güterbock fu anche filologo a Böghazköy.

21 Per queste notizie si veda M. Doğan Alparslan 2001. 
Per comprendere pienamente i criteri che il governo turco applicava nella scelta di accogliere gli studiosi dalla Germania, è esemplare il caso di Emil Forrer.

Venuto a conoscenza della grande apertura della Turchia agli studi di Ittitologia, anche Forrer desiderò trasferirvisi allo scopo di ottenere un insegnamento universitario ${ }^{22}$. Diversamente però da Landsberger e Güterbock, invitati dal governo stesso su segnalazione di un loro giovane studente, Forrer entrò autonomamente in contatto con la diplomazia turca. Ai funzionari giustificò il suo desiderio di trasferirsi in Turchia adducendo ragioni personali e illustrando le restrizioni che, a suo dire, il governo tedesco stava apportando alla disciplina. Ricevuti in un primo momento riscontri positivi alle sue richieste, Forrer si impegnò a fondo nell'elaborazione di un progetto di insegnamento da presentare alle autorità turche. Il progetto era estremamente complesso e articolato prevedendo quattordici lingue antiche che in qualche modo avevano avuto a che fare con l'Anatolia: dal protohattico, all'ittita, al luvio, all'ario, al persiano; erano poi proposti quattro seminari di approfondimenti paleografici e linguistici, tre diversi insegnamenti di storia ittita, divisa per fasi, antica, media, nuova, sei seminari di cultura generale ittita, luvia e hurrita (dalla politica alla religione); ancora, seminari di economia, società, diritto, archeologia. Le autorità turche in un primo momento sembrarono approvare questo piano di lavoro, senza tuttavia formulare alcun impegno concreto. Con l'appoggio di alcuni membri della Türk Tarih Kurumu solo alla fine di febbraio del 1937 Forrer riuscì ad entrare in contatto con il decano della Facoltà di Filosofia di Ankara e la lettera in cui lo studioso illustra dettagliatamente il suo progetto, si chiude con la sua e il saluto nazista 'Heil Hitler!'.

Il piano di Forrer, seppur appoggiato e sponsorizzato dalla Società di Cultura, fu accolto in Turchia con un certo gelo; le notizie che circolarono sottolinearono soprattutto gli elevati costi che la sua applicazione avrebbe comportato, ma non si può escludere che nella decisione di non invitare Forrer rientrassero anche motivazioni politiche. In ogni caso, con l'arrivo ad Ankara di Landsberger e poi di Güterbock nel 1936, fu palese che non vi fosse un concreto interesse nei riguardi di Forrer, che infatti non fu mai chiamato.

Nel frattempo Sedat Alp continuava il suo soggiorno in Germania cercando di imparare il più possibile dagli insegnamenti dei maggiori orientalisti dell'epoca: Hans Ehelolf, Emil Forrer, Bruno Meissner, Adam Falkenstein. L'impegno richiesto era notevole: lo studio era intenso, molti i corsi da seguire; Alp cominciava a studiare alle 5,00 del mattino e continuava per tutto il resto della giornata. L'eccessivo carico di lavoro e la notevole pressione psicologica gli causarono dei problemi di salute, come l'alopecia di cui soffrì in quegli anni. Nel 1940 conseguì il titolo di Dottore di Ricerca in Ittitologia in Germania, diventando il primo studente turco ad essersi addottorato in questa disciplina. Si era intanto giunti al 1941, alle soglie del Secondo Conflitto Mondiale e gli studenti turchi all'estero, Alp compreso, furono richiamati in patria.

Poco dopo il suo rimpatrio, Alp divenne professore di Ittitologia all'Università di Ankara. Intorno al 1948 Güterbock e Landsberger lasciarono la Turchia, forse per l'inasprimento delle relazioni interuniversitarie. Landsberger si trasferì a Chicago, mentre Güterbock si fermò un anno in Svezia, dove insegnò all'Università di Uppsala, per partire

22 Per le notizie su E. Forrer rimandiamo a Oberheid 2007, in particolare 207 e segg. 
anche lui alla volta di Chicago nel 1949, dove poi restò e gettò le basi per il progetto del Chicago Hittite Dictionary, tutt'ora in fieri. Alla loro partenza i due professori lasciarono un'avviata e fiorente scuola di studiosi turchi di alto livello nei vari campi della disciplina.

\section{CONCLUSIONI}

Da questa breve panoramica sulle vicende che coinvolsero direttamente gli studi di Ittitologia e i suoi protagonisti all'epoca della fondazione della Repubblica Turca, è evidente il significativo interesse di Atatürk per questa disciplina, oltre che per gli studi storico-archeologici in generale, a prescindere dalle motivazioni che ne furono alla base. Notevole infatti fu l'impulso che ad essa venne dato, sia in Turchia che all'estero, e gli sviluppi ulteriori che seguirono, i cui effetti positivi, dal punto di vista scientifico, si protraggono ancor oggi.

\section{BIBLIOGRAFIA}

ARAR, I.

1981 Atatürk'ün günümüz olaylarına 1şık tutan bazı konuşmaları (Speeches by Atatürk that shed light on current events): Belleten 45 (1981), pp. 5-26.

ATAKUMAN, C.

2008 Cradle or Crucible. Anatolia and archaeology in the early years of the Turkish Republic (1923-1938): Journal of Social Archaeology 8 (2008), pp. 214-234 (http://jsa.sagepub.com/cgi/content/abstract/8/2/214).

BOSENSKI, C.

$1869 \quad$ Les Turcs anciens et modernes, Costantinopoli 1869.

CAHUN, L.

1896 Introduction Générale à l'Histoire de l'Asie, Turcs et Mongoles, Paris 1896.

ÇAMBEL, H.C.

1932 Ege Tarihinin Menșeine Umumi bir Bakıș (A general look on the roots of the Aegean History): Birinci Türk Tarih Kongresi, Konferanslar Müzakere Zabitlarl, Ankara 1932, pp. 199-214.

DOĞAN ALPARSLAN, M.

2001 Two Capitals, two Adventures: Ankara and Hattusa: M. EMINOĞU - Ş. ŞENTÜRK - N. ÖRKI - E. JEAN (eds.), Boğazköy'den Karatepe'ye: Hititbilim ve Hitit dünyasının keşfi/From Boğazköy to Karatepe. Hittitology and the Discovery of the Hittite World, Istanbul 2001, pp. 72-85.

GRASSI, F.

2008 Atatürk. Il fondatore della Turchia moderna, Roma 2008.

HROZNÝ, B.

1915 Die Lösung des hethitischen Problems: Mitteilungen der Deutschen Orient-Gesellschaft 56 (1915), pp. 17-50.

1917 Die Sprache der Hethiter, ihr Bau und ihre Zugehörigkeit zum indogermanischen İNAN, A.

1930a Vatandaș 'Için Medeni Bilgiler (The Main Tenets of Turkish History), Istanbul 1930.

1930b Türk Tarihinin Ana Hatları (Civil Knowledge for the Fellow Citizens), İstanbul 1930. 
1931 Türk Tarihinin Ana Hatlarl:Methal Kısmı (Introduction to the Main Tenets of Turkish History), İstanbul 1931.

KoşAY, H.Z.

1943 Türk Tarih Kurumu Tarafından Alacahöyükte yaptırılan Hafriyatta elde edilen Neticeler (Results of the Alacahöyük Excavations Conducted by Turkish Historical Association), İkinci Türk Tarih Kongresi, Kongrenin Çalıșmaları ve Kongreye Sunulan Tebliğler, 20-25 Eylül 1937, İstanbul 1943, pp. 21-32.

LOSANO, M.G

2012 Laicismo e pluralismo politico in Turchia dalla fine dell'Impero ottomano a oggi: G. Peces-BARba Martínez et alii, Historia de los derechos fundamentales, Madrid 2012, $\mathrm{http}: / / \mathrm{www}$.dircost.unito.it/dizionario/indice alfabetico.shtml.

OBERHEID, R.

2007 Emil O. Forrer und die Anfänge der Hethitologie. Eine wissenschaftshistorische ÖZER, Y.Z. Biografie, Berlin 2007.

1932 Misır Din ve İlahlarının Türklükle Alakası (The Relationship of Egyptian Religion and Gods to Turkishness), Birinci Türk Tarih Kongresi,Konferanslar Müzakere Zabitları, Ankara 1932, pp. 243-260.

ÖZGÜÇ, T.

1982 Ataturk et l'Archéologie (traduzione di H. Gonnet, Institut Français d'Études Anatoliennes, Mémorial Atatürk. Études d'Archéologie et de Philologie Anatoliennes) Paris 1982. 